Supporting Information for

\title{
Visible or near-infrared Light Self-powered Photodetectors Based on Transparent Ferroelectric Ceramics
}

Geng Huangfu ${ }^{\mathrm{a}}$, Hongyuan Xiao ${ }^{\mathrm{a}}$, Lin Guan ${ }^{\mathrm{a}}$, Haoyin Zhong ${ }^{\mathrm{a}}$, Cheng $\mathrm{Hu}^{\mathrm{b}, \mathrm{c}}$, Zhiwen Shi ${ }^{\mathrm{b}, \mathrm{c}}$, and Yiping Guo $\mathrm{G}^{\mathrm{a}, *}$

${ }^{a}$ State Key Laboratory of Metal Matrix Composites, School of Materials Science and Engineering, Shanghai Jiao Tong University, Shanghai 200240, China

${ }^{\mathrm{b}}$ Key Laboratory of Artificial Structures and Quantum Control (Ministry of Education), Shenyang National Laboratory for Materials Science, School of Physics and Astronomy, Shanghai Jiao Tong University, Shanghai 200240, China

${ }^{c}$ Collaborative Innovation Center of Advanced Microstructures, Nanjing 210093, China.

E-mail:ypguo@sjtu.edu.cn

Tel: 86-21-5473892, Fax: 86-21-34202749 


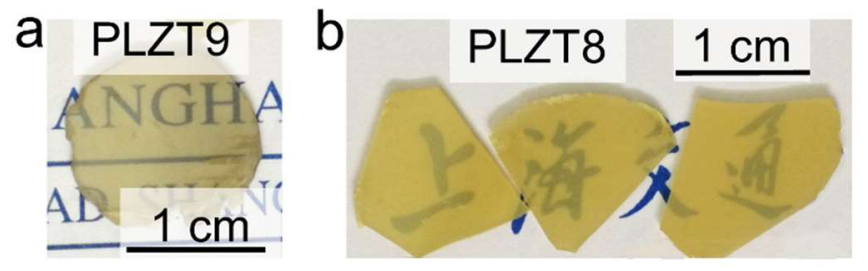

Figure S1. The photos of PLZT9 (a) and PLZT8 (b). The thickness of these samples are about $600 \mu \mathrm{m}$. The characters or patterns behind the samples could be clearly seen, suggesting excellent transmittance to visible light.
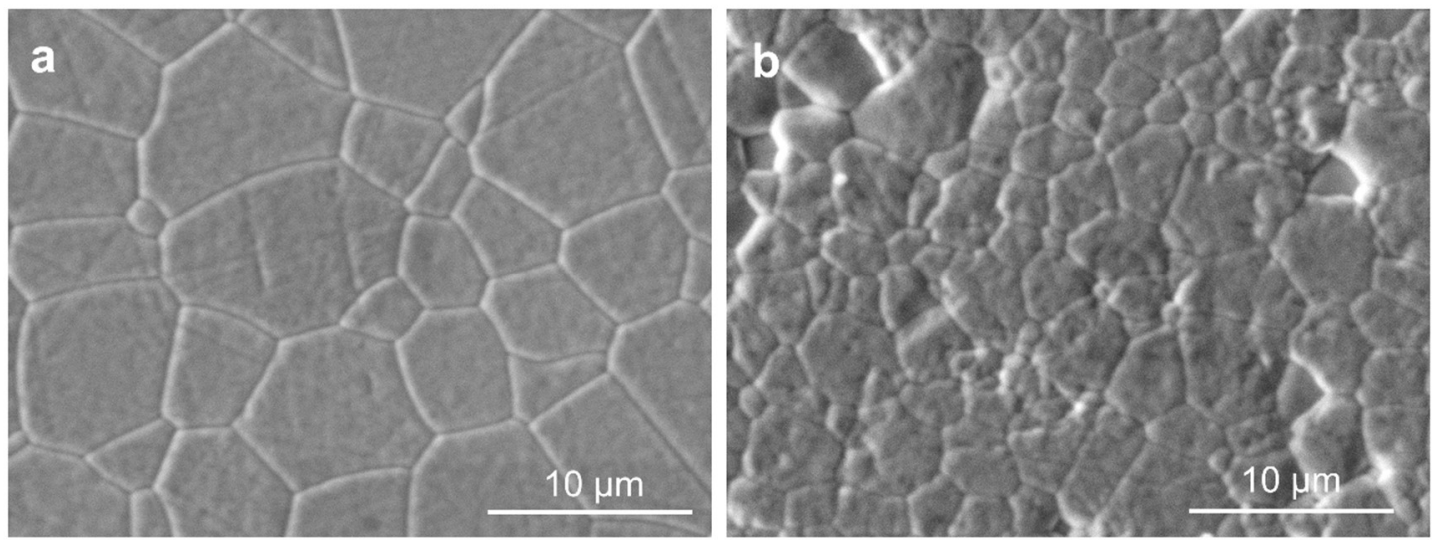

Figure S2. The microstructure of PLZT9 (a) and PLZTN9 (b). These two samples both have high density. The grains of PLZTN9 are obviously smaller than PLZT9, indicating that $\mathrm{Ni}^{2+}$ doping inhibits grain growing. 

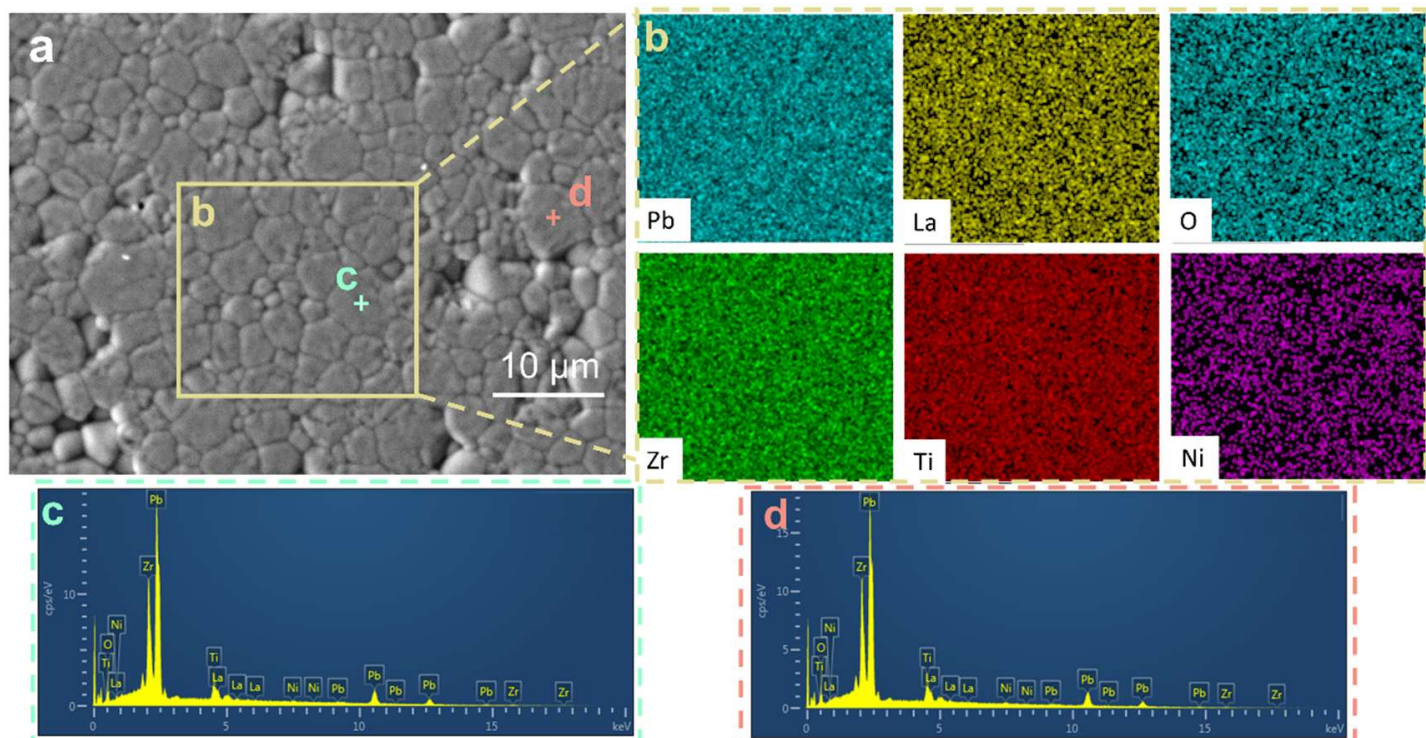

\begin{tabular}{|ccc|}
\hline Element & wt.\% & at. $\%$ \\
\hline $\mathbf{O}$ & 19.18 & 67.47 \\
\hline $\mathbf{T i}$ & 4.94 & 5.80 \\
\hline $\mathbf{N i}$ & 0.47 & 0.45 \\
\hline $\mathbf{Z r}$ & 15.24 & 9.40 \\
\hline $\mathbf{L a}$ & 4.01 & 1.62 \\
\hline $\mathbf{P b}$ & 56.16 & 15.25 \\
\hline Total & 100.00 & 100.00 \\
\hline
\end{tabular}

\begin{tabular}{ccc|}
\hline Element & wt.\% & at.\% \\
\hline $\mathbf{O}$ & 18.52 & 66.48 \\
$\mathrm{Ti}$ & 4.95 & 5.94 \\
\hline $\mathbf{N i}$ & 0.50 & 0.49 \\
\hline $\mathbf{Z r}$ & 15.57 & 9.80 \\
$\mathrm{La}$ & 3.99 & 1.65 \\
$\mathrm{~Pb}$ & 56.46 & 15.65 \\
\hline Total & 100.00 & 100.00 \\
\hline
\end{tabular}

Figure S3. The composition characterization of a micro region of PLZTN9. a) Microstructure of PLZTN9. b) Elemental mapping of selected area in a). c) \& d) Concentration of elements at the corresponding points in a). These results reveal the uniform distribution of $\mathrm{Ni}$ element in grains, and the $\mathrm{Ni}$ concentration close to that of design. 

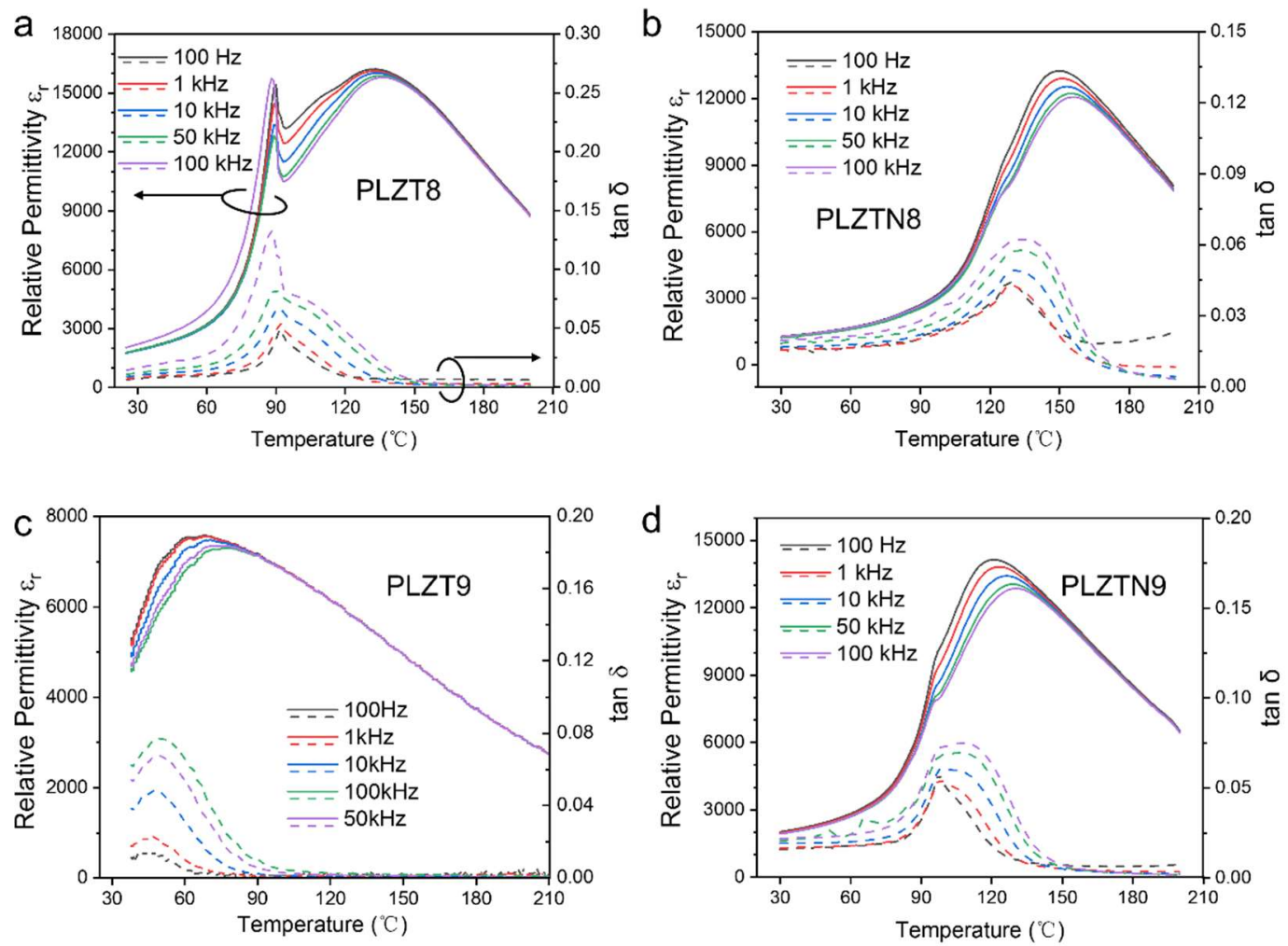

Figure S4. The influence of temperature on relative permittivity $\left(\varepsilon_{r}\right)$ and dielectric loss $(\tan \delta)$ of the four materials in this manuscript. a) PLZT8, b) PLZTN8, c) PLZT9, d) PLZTN9. As marked in figure a, the solid lines are correlated to $\varepsilon_{r}$ and dashed lines are correlated to $\tan \delta$. The temperature at which the $\varepsilon_{r}$ reaches its maximum value is regarded as $T_{m}$. The depolarization behavior of ferroelectric samples (PLZT8, PLZTN8 and PLZTN9) is indicated by a peak on the $\varepsilon_{r}-T$ curve, and happens at depolarization temperature $\left(T_{d}\right)$. In addition, the frequency dispersion of $\varepsilon_{r}$ above $T_{d}$ suggest the characteristic of relaxor ferroelectrics. 

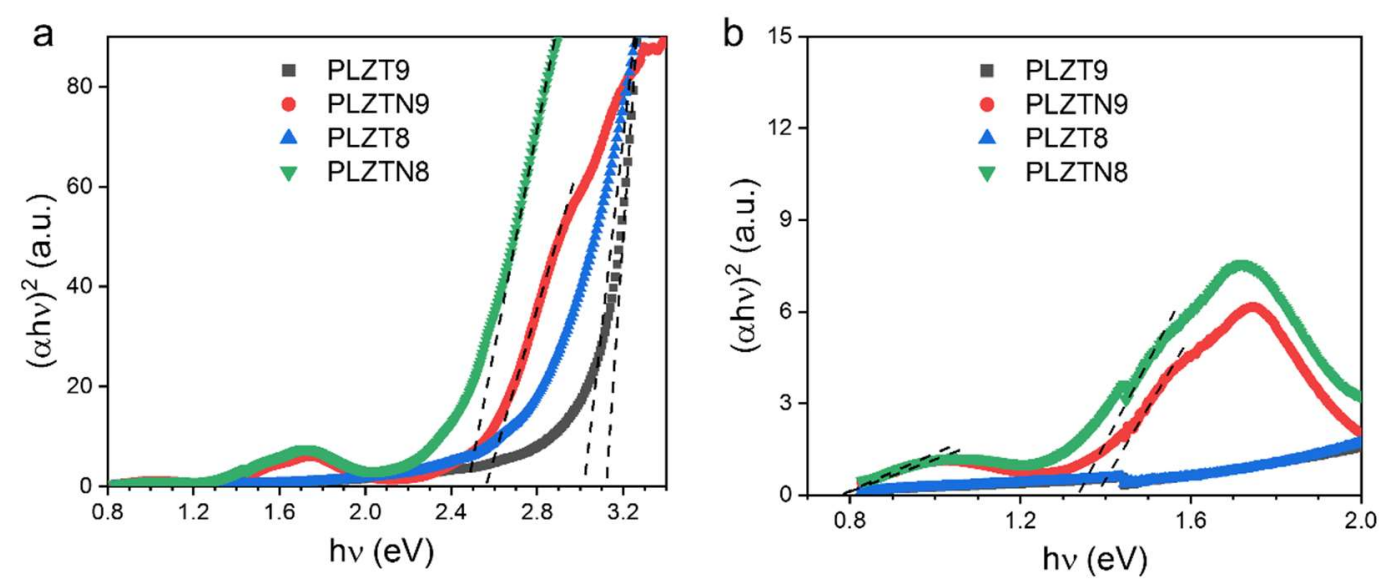

Figure S5. The curves of $(\alpha h v)^{2}$ versus $h v$ of the four samples. a) Illustration of bandgaps of PLZT and PLZTN ceramics. b) A partial enlargement of a), showing the other two possible transitions of electrons in Ni doped samples. The breakpoints at 1.44 $\mathrm{eV}$ are due to instrument jitter. These results suggest that $\mathrm{Ni}^{2+}$ doping can decrease the bandgap of PLZT, and introduce a gap-state between conductive band and valence band.

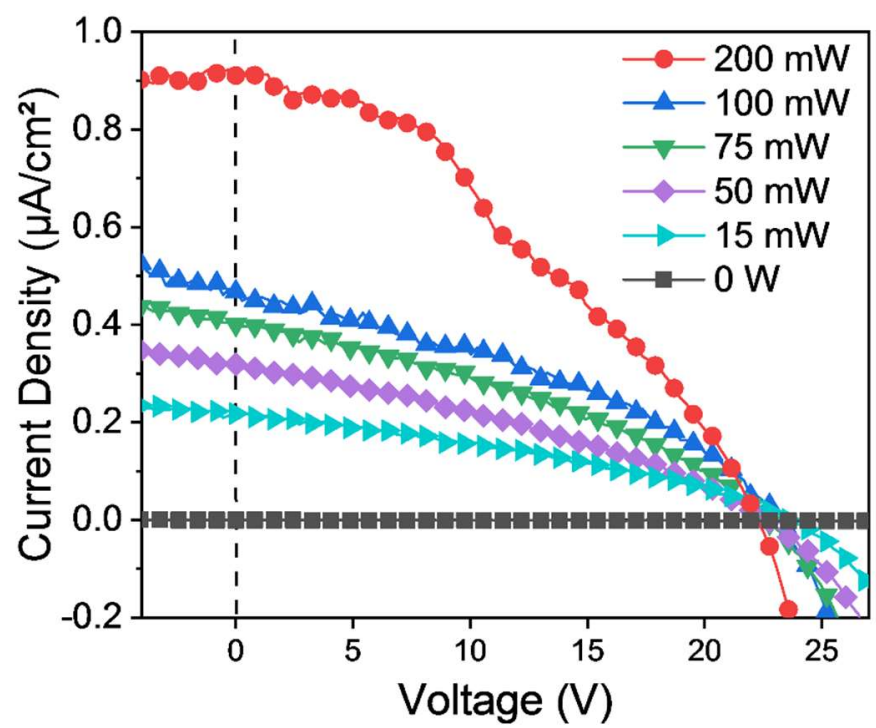

Figure S6. $J-V$ (current density versus voltage) curves of PLZTN9 illuminated by 405 $\mathrm{nm}$ light. It shows very low dark current density, stable open circuit voltage and short circuit current density raising with the increase of light intensity. 

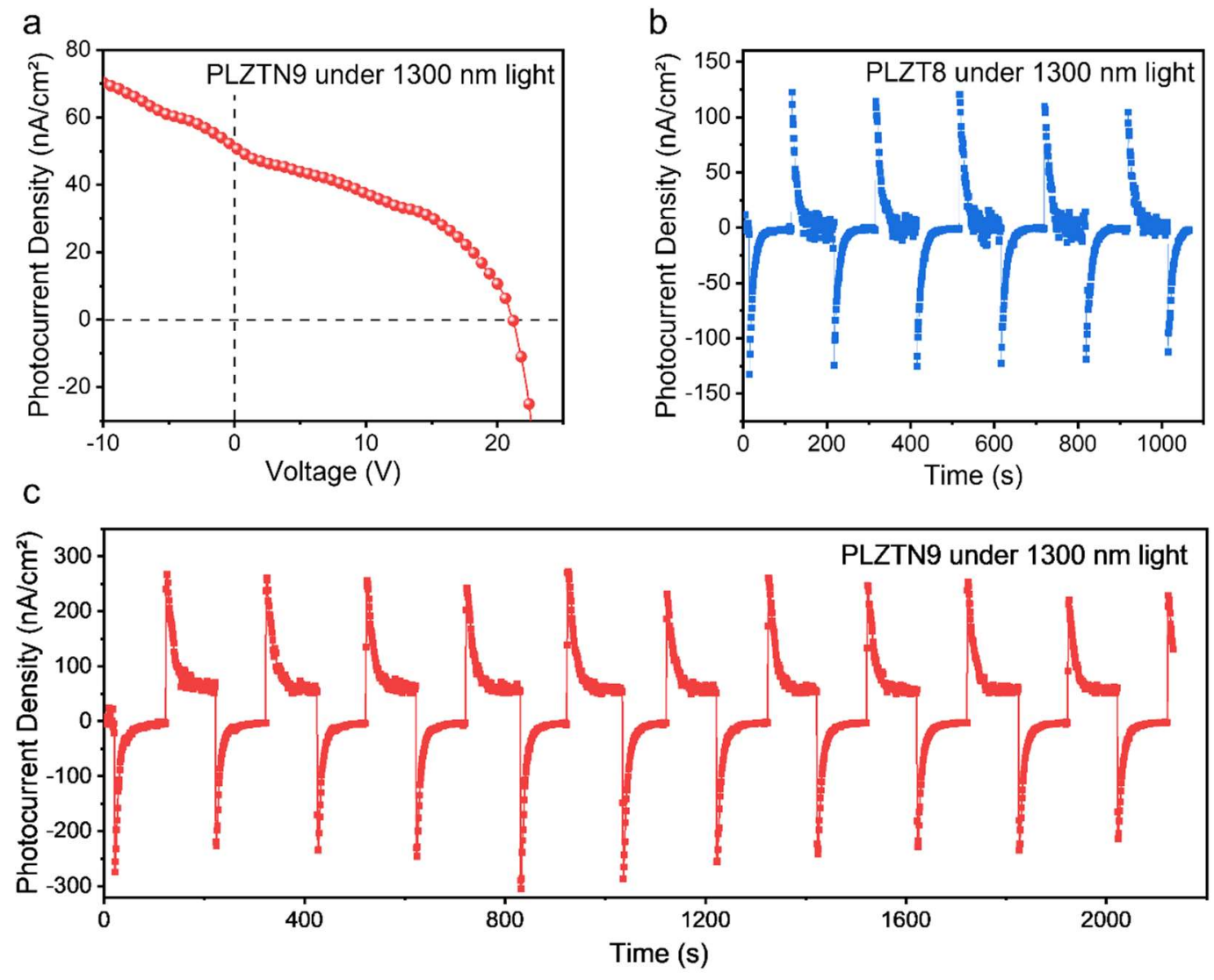

Figure S7. The $J-V$ (PLZTN9) and $J$ - $t$ curves (PLZTN9 and PLZT8) under $1300 \mathrm{~nm}$ light of $100 \mathrm{~mW}$, and the facula area is about $0.36 \mathrm{~cm}^{2}$. a) $J-V$ curve of PLZTN9 under $1300 \mathrm{~nm}$ illumination. b) The light-dark response ( $J$ - $t$ curve) without bias of PLZT8. c) The light-dark response ( $J$ - $t$ curve) without bias of PLZTN9. It is obvious that PLZT8 shows no photovoltaic output but only pyroelectric current under $1300 \mathrm{~nm}$ illumination. On the contrary, PLZTN9 has stable photovoltaic effect stimulated by near infrared (1300 nm) light. 